\title{
OPTICS STUDIES FOR THE T9 BEAM LINE IN THE CERN PS EAST AREA SECONDARY BEAM FACILITY
}

\author{
L. Durieu, M. Martini and A.-S. Müller, CERN
}

\begin{abstract}
The CERN PS East Area provides secondary beams in the momentum range from 1 to $15 \mathrm{GeV} / \mathrm{c}$ for tests of LHC detector components (e.g. for the LHC experiments) and for physics experiments (such as HARP). This latter experiment requires high quality beams of very small size within tight tolerances. In the framework of the HARP beam commissioning, extensive optics studies have been made. Simultaneous measurements of Twiss parameters and emittance were used to obtain a set of initial parameters at the production target. The measurements were consolidated by detailed tracking simulations. This paper gives an overview of the optics design and beam study activities in the T9 beam line of the PS East Area and compares measurements and simulation results.
\end{abstract}

\section{INTRODUCTION}

Since its renovation in 1997 the CERN PS East Area provides secondary beams in the momentum range from 1 to $15 \mathrm{GeV} / \mathrm{c}$ and a primary proton beam of $24 \mathrm{GeV} / \mathrm{c}$. There are two targets present in the area, one feeding three secondary branches, T9, T10 and T11 and one feeding secondary channel T7. Typical intensities are of the order of $10^{6}$ particles per spill of about $300 \mathrm{~ms}$ and per channel. For each secondary line the momentum selection is done with the help of a horizontal collimator in a dispersive region. The beam intensity can be adjusted with a vertical ("intensity") collimator.

\subsection{The Extended T9 Beam Line}

In the framework of the neutrino factory study, several design options are being discussed for the proton driver, ranging from a $2.2 \mathrm{GeV}$ super-conducting $\mathrm{H}^{-}$linac to a $16 \mathrm{GeV}$ rapid-cycling proton synchrotron. To take a decision, the overall pion production yield at the energies in question has to be known to a precision better than the $50 \%$ uncertainty available from current Monte Carlo generators.

The HARP experiment [1] is located in the $90 \mathrm{~m}$ long extended T9 beam line [2] which consists of the original line [3] and an additional quadrupole doublet at the end to cope with the required beam conditions. HARP aims at a $5 \%$ precision of the overall pion yield from a second target located at about $80 \mathrm{~m}$. To achieve this, HARP needs small secondary beam sizes under stable conditions in a momentum range between 1.5 and $15 \mathrm{GeV} / \mathrm{c}$. The particles generated at the first target are mainly charged pions and kaons but the beam of course contains a strong component of scattered protons. In case of selection of positive particles, protons dominate. The positioning of the various

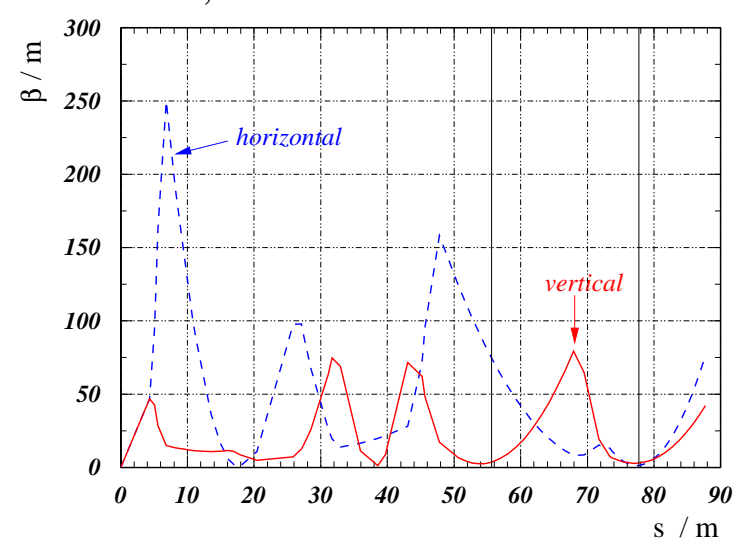

Figure 1: Optics of the T9 secondary beam line: horizontal and vertical $\beta$-function as function of position along the line (initial Twiss parameters derived from geometrical constraints are $\beta_{x, y}=0.4 \mathrm{~m}$ and $\alpha_{x, y}=0$ ). The solid line at about $57 \mathrm{~m}$ denotes the first detector parts, the line at about $78 \mathrm{~m}$ the final focus with the second target.

HARP detector components imposes important constraints on the horizontal and vertical beam size at several places along the line. Further constraints are given by the local aperture of the magnetic elements and the need to have a reasonably small beam at the momentum collimator and profile monitors. Figure 1 shows the horizontal and vertical $\beta$-functions along the T9 beam line.The solid line at about $57 \mathrm{~m}$ denotes the first detector components, the line at about $78 \mathrm{~m}$ the final focus.

Since the T9 beam is generated by a target, Twiss parameters and emittance at the entrance of the line are unknown. The initial design is therefore based on geometrical assumptions for the initial parameters (with a $5 \mathrm{~mm}$ diameter target, considering distance and aperture of the first quadrupole), which are finally determined by the acceptance of the transport channel. Measurements and simulations for this purpose are discussed in detail in the following section.

\section{OPTICS STUDIES}

\subsection{Simulation}

The theoretical acceptance of the line can be determined by extensive tracking. The MAD program [4] was used to propagate a large uniform distribution of initial particle positions through the line (covering an area larger than the target cross-section). Figure 2 shows the resulting horizontal and vertical particle positions as function of distance from the beginning of the line for accepted tracks. The phase space distributions of these particles at the entrance of the line are shown in Fig. 3. The left hand plot shows the horizontal, the right hand plot the vertical phase space representation of the initial coordinates. The ellipses indi- 

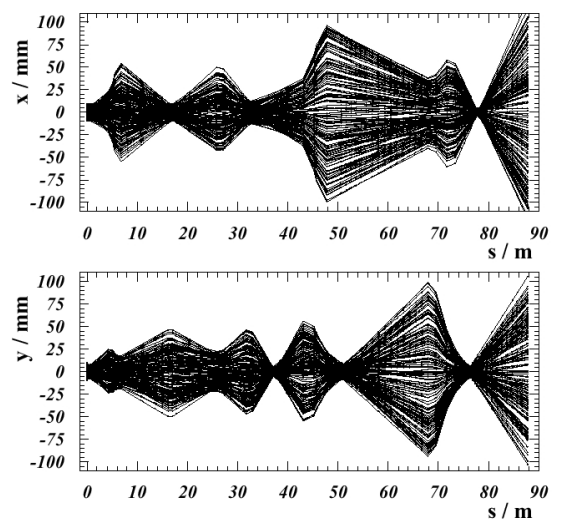

Figure 2: Results of MAD [4] tracking for the T9 beam line. The upper plot shows the horizontal, the lower one the vertical particle position as function of distance from the beginning of the line.

cate the reconstructed initial Twiss parameters and acceptances obtained from the calculation of the equivalent RMS emittance according to $\varepsilon_{R M S}=\sqrt{\left\langle u^{2}\right\rangle\left\langle u^{\prime 2}\right\rangle-\left\langle u u^{\prime}\right\rangle^{2}}$ with $\alpha=-\left\langle u u^{\prime}\right\rangle / \varepsilon_{R M S}$ and $\beta=\left\langle u^{2}\right\rangle / \varepsilon_{R M S}$, where $u$ stands for the horizontal or vertical plane and providing that $\langle u\rangle$ and $\left\langle u^{\prime}\right\rangle$ vanish. Statistical uncertainties are given, derived from higher order moments of the distribution. The acceptances obtained by tracking are $a_{x}=(12.7 \pm 0.3) \mu \mathrm{m}$ and $a_{y}=(15.1 \pm 0.3) \mu \mathrm{m}$. The initial parameters derived from the theoretical acceptance are $\alpha_{x}=0.22 \pm 0.03, \alpha_{y}=0.62 \pm 0.04, \beta_{x}=(1.79 \pm 0.05) \mathrm{m}$ and $\beta_{y}=(1.87 \pm 0.05) \mathrm{m}$. These $\beta$-functions are obviously larger than those calculated from geometrical constraints (see Fig. 1) but show the same aspect ratio.

\subsection{Measurement}

Simultaneous measurements of Twiss parameters and emittance were performed with "scans" of quadrupole gradients. In this method, the beam size is measured with profile monitors (multi wire proportional chambers, MWPCs, in the case of T9) as function of the quadrupole gradient. The beam size at a given point is described by $\sigma_{i, 11}^{2}=C_{i}^{2} c_{11}^{b}+2 S_{i} C_{i} c_{12}^{b}+S_{i}^{2} c_{22}^{b}$, where $C_{i}$ and $S_{i}$ are elements of the transformation matrix from the point of measurement to the point of interest, the $c_{i j}^{b}$ are elements of the beam matrix at the point of interest and the $\sigma_{i, 11}$ are the
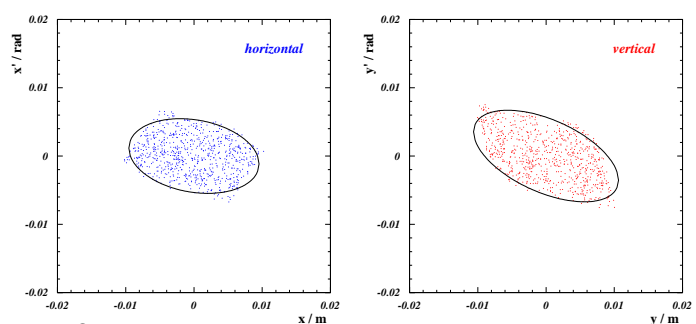

Figure 3: Horizontal (left) and vertical (right) phase space representation of accepted tracks (759 from 24000 generated). From these distributions, Twiss parameters at the target at the entrance of the line can be approximated, which are indicated by the ellipses.

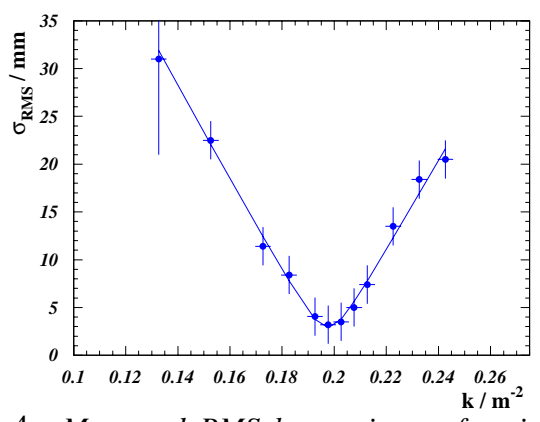

Figure 4: Measured RMS beam size as function of normalised quadrupole gradient $k$. The filled circles with error bars represent the measured beam sizes, the curve corresponds to the fit.

measured beam sizes. Therefore these scans can be used to extract the beam matrix elements by a fit, and extrapolation to the entrance of the line. Figure 4 shows such a scan. The filled circles with error bars represent the measured beam sizes, the curve corresponds to the fit. The beam emittances extracted from these scans are $\varepsilon_{x}=(10.5 \pm 1.1) \mu \mathrm{m}$ and $\varepsilon_{y}=(3.9 \pm 1.4) \mu \mathrm{m}$. The corresponding Twiss parameters were measured to be $\alpha_{x}=-(0.4 \pm 0.6), \alpha_{y}=2.2 \pm 4.3$, $\beta_{x}=(0.8 \pm 0.4) \mathrm{m}$ and $\beta_{y}=(0.9 \pm 1.1) \mathrm{m}$. Additional systematic uncertainties arise from the non-Gaussian shape of the beam which is caused by collimation. This might prevent the beam filling the phase space ellipse completely and therefore alter the determination of the Twiss parameters.

\section{OPERATIONAL ASPECTS}

The operation of the HARP experiment requires comparable beam conditions over the full momentum range. This is made difficult by the increase in multiple Coulomb scattering at lower momenta. Several detectors are placed in the beam line (for example time-of-flight scintillators) and the beam has to pass through air. This leads to an emittance blow-up caused by multiple scattering. This emittance increase is given by [5]

$$
\Delta \varepsilon=\frac{1}{2}\left\langle\theta^{2}\right\rangle\left[\beta_{0}+L \alpha_{0}+\frac{L^{2}}{3} \gamma_{0}\right]
$$

where $\alpha_{0}, \beta_{0}$ and $\gamma_{0}$ are the Twiss parameters at the scatterer, $L$ the thickness of the obstacle and $\sqrt{\left\langle\theta^{2}\right\rangle}$ the RMS

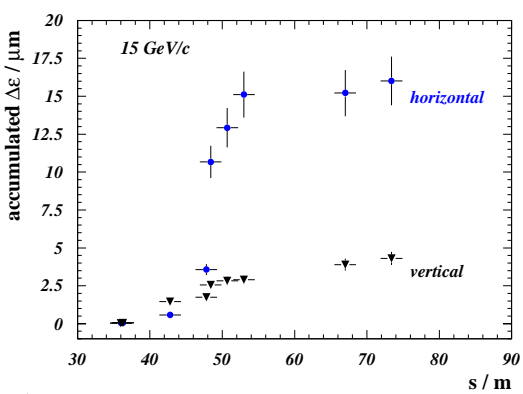

Figure 5: Estimated accumulated (normalised) emittance blow-up $(\Delta \varepsilon)$ as function of position in the beam line. Horizontal and vertical emittance blow-up are shown for a momentum of $15 \mathrm{GeV/c}$. 

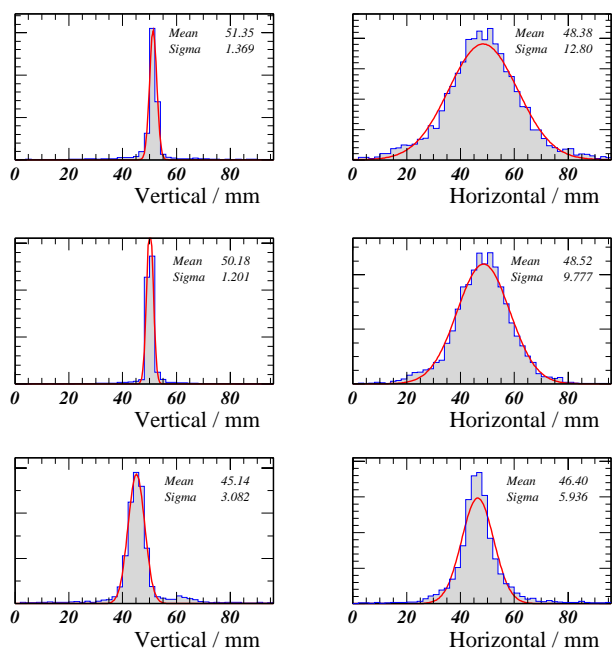

$\varepsilon_{V}=1.95$

$\varepsilon_{H}=10.06$

Figure 6: Beam profiles measured with the three HARP beam chambers kindly provided by $P$. Gorbounov of the HARP collaboration. The left hand column shows vertical, the right hand column horizontal beam profiles. The threefold measurement allows an on-line estimation of the emittance (quoted below the plots) which is in reasonable agreement with the quadrupole scan measurements.

scattering angle [6]

$$
\sqrt{\left\langle\theta^{2}\right\rangle}=13.6 z \frac{1}{\beta p} \sqrt{\frac{L}{X_{0}}}\left\{1+0.038 \ln \frac{L}{X_{0}}\right\} .
$$

In this equation, $X_{0}$ is the radiation length, $z, p$ and $\beta$ are the charge state, total momentum (in $\mathrm{MeV} / \mathrm{c}$ ) and velocity of the incident ion relative to the speed of light. Figure 5 shows the estimated accumulated (normalised) emittance blow-up $(\Delta \varepsilon)$ as function of position in the beam line. Horizontal and vertical emittance blow-up are shown for a momentum of $15 \mathrm{GeV} / \mathrm{c}$. The significant increase in horizontal emittance due to scattering in a high- $\beta$ region is clearly visible. As a consequence, the amount of material in the beam path was reduced and the most disturbing scatters moved to a place of lower $\beta$. As a result, the beam size contribution from scattering was reduced by about a factor two.

To survey beam quality and steering, the HARP experiment is equiped with its own MWPCs. Figure 6 shows beam profiles measured with these chambers. The left hand column shows vertical, the right hand column horizontal beam profiles. This threefold measurement allows an on-line estimation of the emittance using the known transfer matrix. The quoted result is in reasonable agreement with the results obtained by quadrupole scans. Figure 7 finally shows the beam size as function of momentum measured in 2000 and in 2001. The upper plot shows the horizontal, the lower plot the vertical RMS beam size. The success of repeated beam tuning sessions and the relocation of detectors causing emittance increase by multiple Coulomb scattering is clearly visible.

\section{SUMMARY}

The original T9 beam line has been extended by adding one quadrupole doublet to meet the tight beam size requirement
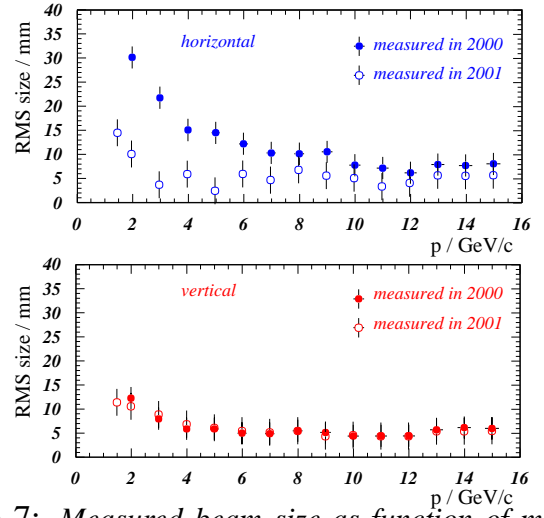

Figure 7: Measured beam size as function of momentum. The upper plot shows the horizontal, the lower plot the vertical RMS beam size. The filled symbols correspond to measurements done in 2000, the open symbols to measurements of 2001. The increase in beam size for lower momentum due to multiple Coulomb scattering is clearly visible.

of the HARP experiment, especially at low momenta where the beam suffers the harmful effect of multiple scattering. Additional constraints such as apertures and the limited strength of existing quadrupoles need also to be considered. The design of the new T9 optics has been verified by extensive tracking using Monte Carlo techniques to derive the acceptance of the channel. Monte Carlo simulations and classical optics design, based on matching with geometrical assumptions for initial parameters, yield both symmetric beta-functions at the entry of T9 line. Experimental results obtained during the commissioning phase of the beam line, after fine tuning and better positioning of some equipment along the line, showed that the beam sizes at all the requested momenta remain within the tolerances of the HARP experiment.

\section{ACKNOWLEDGEMENTS}

Sincere thanks to all who have contributed to the results presented here and especially to J.-Y. Hémery, J.-P. Riunaud and L. Zani for interesting and instructive discussions.

\section{REFERENCES}

[1] M. Catanesi et al. Proposal to study hadron production for the neutrino factory and for the atmospheric neutrino flux. CERN-SPSC 99-35, CERN, November 1999.

[2] L. Durieu, M. Giovannozzi, M. Martini, L. Zani. Design of an Extension of the T9 Beam Line for Hadron Production Study. PS/CA 99-027, CERN, 1999.

[3] L. Durieu, O. Ferrando. Design of T9 (Atlas/CMS) for EHNL. PS/PA 96-39, CERN, 1996.

[4] H. Grote and F. C. Iselin. The MAD Program, User's Reference Manual. SL Note 90-13 (AP) (Rev. 4), CERN, March 1995.

[5] A. Müller. Description of Beam-Matter Interaction in the Covariance Matrix Formalism - Application to Modification of Emittance and Twiss Parameter. CERN/PS 2001-013 (AE), CERN, May 2001.

[6] D.E. Groom et al. Review of Particle Physics. In Eur. Phys. J. C 15. 2000. 